\title{
Diet and deprivation in pregnancy
}

\author{
Paul Haggarty ${ }^{1,2 *}$, Doris M. Campbell ${ }^{2}$, Susan Duthie ${ }^{1}$, Katherine Andrews ${ }^{1}$, Gwen Hoad ${ }^{1}$, \\ Chandrika Piyathilake ${ }^{3}$ and Geraldine McNeill ${ }^{4}$ \\ ${ }^{1}$ Nutrition and Epigenetics Group, Rowett Institute of Nutrition and Health, University of Aberdeen, Greenburn Road, Bucksburn, \\ Aberdeen AB21 9SB, UK \\ ${ }^{2}$ Department of Obstetrics and Gynaecology, University of Aberdeen, Aberdeen AB9 2ZD, UK \\ ${ }^{3}$ Division of Nutritional Biochemistry and Genomics, University of Alabama, Birmingham, AL 35294-3360, USA \\ ${ }^{4}$ Population Health Section, University of Aberdeen, Aberdeen AB25 2ZD, UK
}

(Received 17 October 2008 - Revised 17 April 2009 - Accepted 22 April 2009 - First published online 17 August 2009)

Deprivation is associated with poor pregnancy outcome but the role of nutrition as a mediating factor is not well understood. We carried out a prospective cohort study of 1461 singleton pregnancies in Aberdeen, UK during 2000-6. We measured nutrient intake and supplement use, B vitamin and homocysteine status, birth weight, gestational age, neonatal treatment and socio-economic deprivation status. Women in the most deprived deciles were approximately 6 years younger and half as likely to take folic acid supplements periconceptually as the least deprived mothers. Deprivation was associated with low blood folate, high homocysteine and diets low in protein, fibre and many of the vitamins and minerals. The diets of the more deprived women were also characterised by low intakes of fruit, vegetables and oily fish and higher intakes of processed meat, fried potatoes, crisps and snacks. Deprivation was related to preterm birth (OR $1.14(95 \%$ CI 1.03, 1.25); $P=0.009)$ and whether the baby required neonatal treatment (OR 1.07 (95\% CI 1.01, 1.14); $P=0.028)$. Low birth weight was more common in women consuming diets low in vitamin C (OR 0.79 (95\% CI 0.64, 0.97); $P=0.028$ ), riboflavin (OR 0.77 (95\% CI 0.63, 0.93); $P=0.008$ ), pantothenic acid (OR 0.79 (95\% CI 0.65, 0.97); $P=0.023)$ and sugars (OR 0.78 (95\% CI 0.64, 0.96); $P=0.017$ ) even after adjustment for deprivation index, smoking, marital status and parity. Deprivation in pregnancy is associated with diets poor in specific nutrients and poor diet appears to contribute to inequalities in pregnancy outcome. Improving the nutrient intake of disadvantaged women of childbearing age may potentially improve pregnancy outcome.

Pregnancy: Diet: Deprivation: Folic acid: Birth weight: Preterm birth: Social class

Pregnancy is acknowledged as a key life stage critical for both the current and the next generation ${ }^{(1)}$. Low birth weight is associated with poor obstetric outcome and long-term disadvantage including reduced stature ${ }^{(2)}$, poor cognitive function $^{(3,4)}$ and increased risk of $\mathrm{CVD}^{(5)}$. Low birth weight is more common in deprived areas of Britain ${ }^{(6)}$ and low social class is associated with increased risk of low birth weight and perinatal mortality ${ }^{(7-10)}$. Indeed, it has been estimated that more than one-quarter of low birth weight is attributable to social inequalities ${ }^{(11)}$ and there is little evidence that the situation is improving. Social inequalities in low birth weight, preterm delivery and low birth weight for gestational age decreased throughout the 1980s in Scotland but increased again between 1991 and 2000 to levels similar to those found 20 years previously $^{(7,8,12)}$.

Smoking is known to account for some of the social inequality in birth weight but nutrition is also thought to be a causal factor and the Acheson report ${ }^{(1)}$ highlighted the importance of diet in policies aimed at improving health and reducing health inequalities in women of childbearing age and expectant mothers. Many UK health policies are aimed at the elimination of food poverty and improvement of the diet in poorer socio-economic groups ${ }^{(13)}$. These range from the Welfare Food Scheme, designed to protect children's health during rationing, to the recently introduced Healthy Start programme through which pregnant women from lowincome families receive free fruit and vegetables and vitamin supplements. Poor folate status in women of childbearing age, particularly from the more deprived groups, also prompted the recent recommendation that all flour in the UK be fortified with folic acid in order to address the problem of neural tube defect ${ }^{(14)}$.

There is a need to understand the interaction between nutrition and deprivation in pregnancy and their relationship to pregnancy outcome. The aims of the present study were to evaluate current nutrient intake and status in pregnancy in relation to a high-resolution measure of multiple deprivation in a Scottish population. Particular attention was given to the $\mathrm{B}$ vitamins.

\section{Materials and methods}

The study was approved by the Grampian Research Ethics Committee and all women in the study gave informed written 
consent to take part. Women who were diabetic, carrying multiple pregnancies, who conceived as a result of fertility treatment, or in whom clinical data were not available, were excluded. Of 1461 eligible women, 1277 were enrolled sequentially (during 2000-6) at Aberdeen Maternity Hospital when attending for ultrasound scan; a further 184 were recruited later in pregnancy. Information on each pregnancy was abstracted from the Aberdeen Maternity and Neonatal Databank and the data anonymised before linking to the dietary and laboratory data.

Low birth weight was defined as less than $2500 \mathrm{~g}$ or the lowest decile for the standardised birth weight score (birth weight $z$-score adjusted for gestational age, sex and parity) ${ }^{(15)}$. The main advantage of this measure over an absolute birth weight cut-off is that it allows the effect of preterm delivery to be considered separately. Any delivery that occurred before 37 weeks' gestation was defined as preterm. Pregnancies in which the newborn was admitted to the neonatal unit for any reason were categorised as requiring neonatal treatment. Deprivation was assessed using the Scottish Index of Multiple Deprivation 2004 ${ }^{(16)}$. This metric provides a comprehensive picture of multiple deprivation by area (postcode) based on thirty-one indicators which describe various aspects of deprivation within six domains of current income, employment, housing, health, education, skills and training and geographic access to services and telecommunications. The deciles are based on the whole population.

Women provided a blood sample at Aberdeen Maternity Hospital at approximately 19 weeks' gestation. Total plasma folate and plasma vitamin $\mathrm{B}_{12}$ were determined by RIA using the Simultrac Radioassay Kit vitamin $\mathrm{B}_{12}\left[{ }^{57} \mathrm{Co}\right] /$ folic acid $\left[{ }^{125} \mathrm{I}\right]$ (MP Biomedicals, Irvine, CA, USA). Plasma total homocysteine (tHcy) was measured by reverse-phase HPLC using a DS30 Hcy Homocysteine Assay Kit and DS30 analyser system (Drew Scientific, Barrow-in-Furness, Cumbria, UK). Total erythrocyte folate was measured using the ninety-six-well plate adaptation of the Lactobacillus casei microbiological assay ${ }^{(17)}$.

Nutrient intake was determined at 19 weeks by a selfadministered FFQ developed for use in Scottish populations ${ }^{(18)}$ designed to provide an estimate of habitual diet. The validity of this questionnaire for ranking intake of nutrients was assessed in forty non-pregnant women aged 19-50 years. Spearman correlation coefficients between intake from the FFQ and from $4 \mathrm{~d}$ weighed diet (subjects were asked to record the description and weight of all the food and drink they consumed over a period of $4 \mathrm{~d}$, including three weekdays and one weekend day) were above 0.4 and mostly above 0.5 for all macronutrients and all minerals and vitamins apart from $\mathrm{Na}, \mathrm{Cl}, \mathrm{Se}$, iodine, vitamin $\mathrm{B}_{12}$, retinol and vitamin $\mathrm{D}$ (all $0 \cdot 25-0 \cdot 39$ ). For thiamine no correlation was found but this is likely to reflect the fact that the $4 \mathrm{~d}$ diet diary was not long enough to capture variation in habitual intake of mycoprotein meat substitutes that are extremely rich in thiamine. The questionnaire, which covers 170 different food items and twenty different food groups, is semi-quantitative; it asks subjects to estimate both the number of times per week they consume each food and the number of measures of food they consume on each of these occasions. Nutrient intakes from natural foods are calculated using the UK
National Nutrient Databank (based on the fifth edition of McCance \& Widdowson's The Composition of Foods and related supplementary volumes). The energy-adjusted nutrient intake provides an estimate of the nutrient density of the diet. Additional questions relating to the use of food supplements (type or brand, amount per d, timing and duration of consumption in relation to stage of pregnancy), dietary restrictions and habits are also included in the questionnaire. A database of the composition of over 300 different supplement products was developed 'in house' to estimate intake from these sources. The supplement database was continually updated as and when new products were recorded by mothers. The nutrient content of the supplements was as recorded by the manufacturer on the product and therefore does not take into account 'overage' or other variations due to factors such as shelf life. The FFQ was designed to provide data on nutrients rather than food groups but it can be used to provide dimensionless information on relative differences within a population. The $z$-score for the intake of the main food groups was calculated and related to deprivation index.

Statistical analysis was carried out using STATA/SE (version 10.0; StataCorp LP, College Station, TX, USA). Multiple linear regression was used for continuous dependent variables (metabolite concentrations, nutrient intake) and logistic regression for categorical outcomes (for example, low birth weight, preterm birth), with adjustment for appropriate covariates.

\section{Results}

The study group characteristics are shown in Table 1. The characteristics of the group were checked against information on all singleton births in Aberdeen Maternity and Neonatal Database over a similar period (data not shown). The group

Table 1. Study group characteristics and pregnancy outcomes ( $n$ 1461) (Mean values and standard deviations, percentages or numbers)

\begin{tabular}{|c|c|c|}
\hline & Mean & SD \\
\hline \multicolumn{3}{|l|}{ Maternal characteristics } \\
\hline Age at delivery (years) & $30 \cdot 4$ & $5 \cdot 3$ \\
\hline Height $(\mathrm{cm})$ & $164 \cdot 1$ & $6 \cdot 7$ \\
\hline Primiparous (\%) & \multicolumn{2}{|c|}{$48 \cdot 9$} \\
\hline Smoker (\%) & \multicolumn{2}{|c|}{$15 \cdot 2$} \\
\hline \multicolumn{3}{|l|}{ SIMD quintile (\%) } \\
\hline 1st & \multicolumn{2}{|c|}{50} \\
\hline 2nd & \multicolumn{2}{|c|}{24} \\
\hline 3rd & \multicolumn{2}{|c|}{10} \\
\hline 4th & \multicolumn{2}{|c|}{10} \\
\hline 5th & \multicolumn{2}{|c|}{5} \\
\hline \multicolumn{3}{|l|}{ Pregnancy outcome } \\
\hline \multicolumn{3}{|l|}{ Baby sex (\%) } \\
\hline Male & & \\
\hline Female & & \\
\hline Gestational age at delivery (weeks) & $39 \cdot 4$ & 1.9 \\
\hline Preterm delivery (<37 weeks) (\%) & \multicolumn{2}{|c|}{5} \\
\hline Birth weight $(g)$ & 3452 & 560 \\
\hline Admitted to neonatal unit (\%) & \multicolumn{2}{|c|}{15} \\
\hline \multicolumn{3}{|l|}{ Pregnancy loss, $n$} \\
\hline Stillbirth & \multicolumn{2}{|c|}{2} \\
\hline Neonatal death in first week & \multicolumn{2}{|c|}{4} \\
\hline Miscarriage & \multicolumn{2}{|c|}{1} \\
\hline
\end{tabular}

SIMD, Scottish Index of Multiple Deprivation. 
was representative of singleton pregnancies in Aberdeen with respect to the key indicators of maternal weight, height, pregnancy complications, birth weight and birth outcome. The frequency of smoking was low $(<10 \%)$ in the least deprived groups with little change across the first four deciles (Fig. 1). Higher levels of deprivation were related to smoking behaviour, with the highest rates of smoking observed in the most deprived groups. Age at delivery and the presence of a partner (married or cohabiting) were also related to level of deprivation with the most deprived mothers being about 6 years younger and almost twice as likely to be without a partner as the least deprived mothers.

Analysis of non-nutritional factors in relation to pregnancy outcome (Table 2) indicated that deprivation was related to preterm birth (OR 1.14 (95\% CI 1.03, 1.25); $P=0.009)$ and whether the newborn required neonatal treatment (OR 1.07 (95\% CI 1.01, 1.14); $P=0.028)$. The risk of low birth weight $(<2500 \mathrm{~g})$ was also related to deprivation (data not shown) but this appeared to be largely due to an effect on preterm birth as the gestation age-adjusted standardised birth weight score was not significantly related to deprivation. Smoking significantly increased the risk of low birth weight even with adjustment for deprivation status (OR 2.87 (95\% CI $1.88,4.40) ; P<0.001)$. Greater age at delivery also increased the risk of neonatal treatment (OR 1.04 (95\% CI $1.01,1.07) ; P=0.012)$. These analyses controlled for marital status and parity, the latter of which was significantly related to the probability of preterm birth (OR 0.43 (95\% CI 0.26 , $0.72) ; P=0.001)$ and neonatal treatment (OR $0.38(95 \% \mathrm{CI}$ $0.28,0.53) ; P<0.001)$, though again the link between parity and low birth weight was primarily a function of preterm birth as the effect was not present after adjustment for gestational age or when using the standardised birth weight score. The significant relationships in Table 2 remained so after adjustment for nutrient intake.

About $40-50 \%$ of the least deprived women reported taking folic acid supplements periconceptually but the women in the most deprived deciles were about half as likely to take supplements at this time (Fig. 1). By week 12 of pregnancy the difference in reported intake by level of
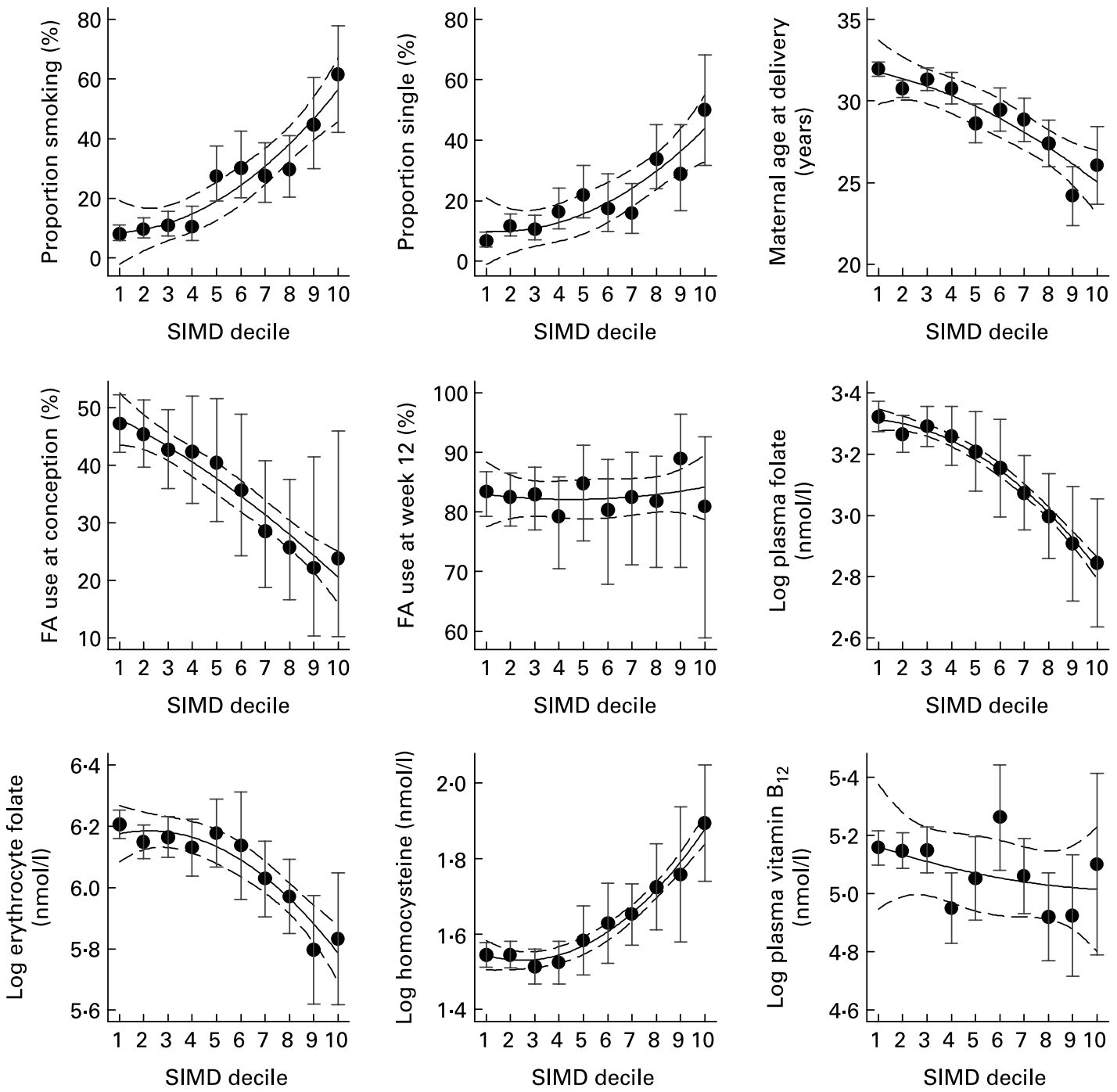

Fig. 1. Trends (polynomial fit $(-)$ with $95 \% \mathrm{Cl}(---)$ ) by Scottish Index of Multiple Deprivation (SIMD) decile for proportions smoking, single, and taking folic acid (FA) supplements periconceptually or within the first 12 weeks of pregnancy (week 12). Maternal age at delivery is also shown together with blood folate, vitamin $\mathrm{B}_{12}$ and homocysteine concentration (log transformed). Values are means, with $95 \% \mathrm{Cl}$ represented by vertical bars. 
Table 2. Effect of non-nutritional factors on pregnancy outcome: risks of low birth weight, preterm delivery and whether the newborn required neonatal care*

(Odds ratios and $95 \%$ confidence intervals)

\begin{tabular}{|c|c|c|c|c|c|c|c|c|c|}
\hline \multirow[b]{2}{*}{ Parameter (OR units) } & \multicolumn{3}{|c|}{$\begin{array}{l}\text { Low birth weight (lowest SBS } \\
\text { decile) }\end{array}$} & \multicolumn{3}{|c|}{ Preterm delivery $(<37$ weeks) } & \multicolumn{3}{|c|}{ Neonatal treatment } \\
\hline & OR & $95 \% \mathrm{Cl}$ & $P$ & OR & $95 \% \mathrm{Cl}$ & $P$ & OR & $95 \% \mathrm{Cl}$ & $P$ \\
\hline SIMD (decile) & 0.98 & $0.91,1.06$ & 0.623 & $1 \cdot 14$ & $1.03,1.25$ & 0.009 & 1.07 & $1.01,1.14$ & 0.028 \\
\hline Smoking category & $2 \cdot 87$ & $1.88,4.40$ & $<0.001$ & 1.39 & $0.74,2 \cdot 60$ & 0.301 & 0.89 & $0.57,1.38$ & 0.591 \\
\hline Age at delivery (years) & 1.00 & $0.96,1.03$ & 0.815 & 1.05 & $1 \cdot 00,1 \cdot 10$ & 0.068 & 1.04 & $1.01,1.07$ & 0.012 \\
\hline Marital status & 1.20 & $0.73,1.97$ & 0.482 & 1.01 & $0.51,2.03$ & 0.970 & 0.87 & $0.55,1.37$ & 0.540 \\
\hline Parity & 1.05 & $0.72,1.51$ & 0.815 & 0.43 & $0.26,0.72$ & 0.001 & 0.38 & $0.28,0.53$ & $<0.001$ \\
\hline
\end{tabular}

SBS, standardised birth weight score; SIMD, Scottish Index of Multiple Deprivation.

${ }^{*}$ Results of logistic regression model with five variables: SIMD decile; smoking in pregnancy category - smoker (coded 1) v. non-smoker (coded 0); marital status - married or cohabiting (coded 1$) v$. divorced or single (coded 0 ); parity - one or more previous pregnancy (coded 1$) v$. none (coded 0 ); age at delivery (continuous variable).

deprivation disappeared and about $80 \%$ of women reported taking folic acid. However, blood folate status measured at 19 weeks showed strong stratification by level of deprivation, with both plasma and erythrocyte folate following the same pattern of a shallow fall in the first three to four deciles followed by a steep decline with increasing level of deprivation. There was a complementary rise in homocysteine concentration with increasing level of deprivation but no change in plasma vitamin $B_{12}$ status.

Regression of energy-adjusted nutrient intake $z$-score on deprivation decile allows comparison on the same graph of a standardised unit change in intake per deprivation decile across all the nutrients (Fig. 2). The diet of the more deprived women was characterised by a low density of protein, fibre and almost all of the vitamins and minerals. The main exceptions were vitamin $\mathrm{A}$ and vitamin $\mathrm{E}$, which showed no stratification by deprivation category, and $\mathrm{Na}$ and $\mathrm{Cl}$ for which the intake was higher in the most deprived women (though not statistically significant), largely reflecting a higher intake of saltrich foods. The diets of the more deprived women also tended to be higher in sugars, starch and fats (with the exception of polyunsaturated fats) but these differences were not statistically significant. Alcohol intake fell with increasing level of deprivation. The generally poorer nutrient intakes associated with deprivation were consistent with food choices: the diet of the more deprived women was characterised by low intakes of fruit and vegetables and higher intakes of fried potatoes, crisps and snacks. Deprived women also consumed more processed meat but less unprocessed meat. Intakes of non-oily fish varied little with level of deprivation but the intake of oily fish was lower in deprived women (Fig. 3).

Such linear regression analysis provides a useful overall summary but for many nutrients the pattern of response to level of deprivation was more complicated and this can be seen in

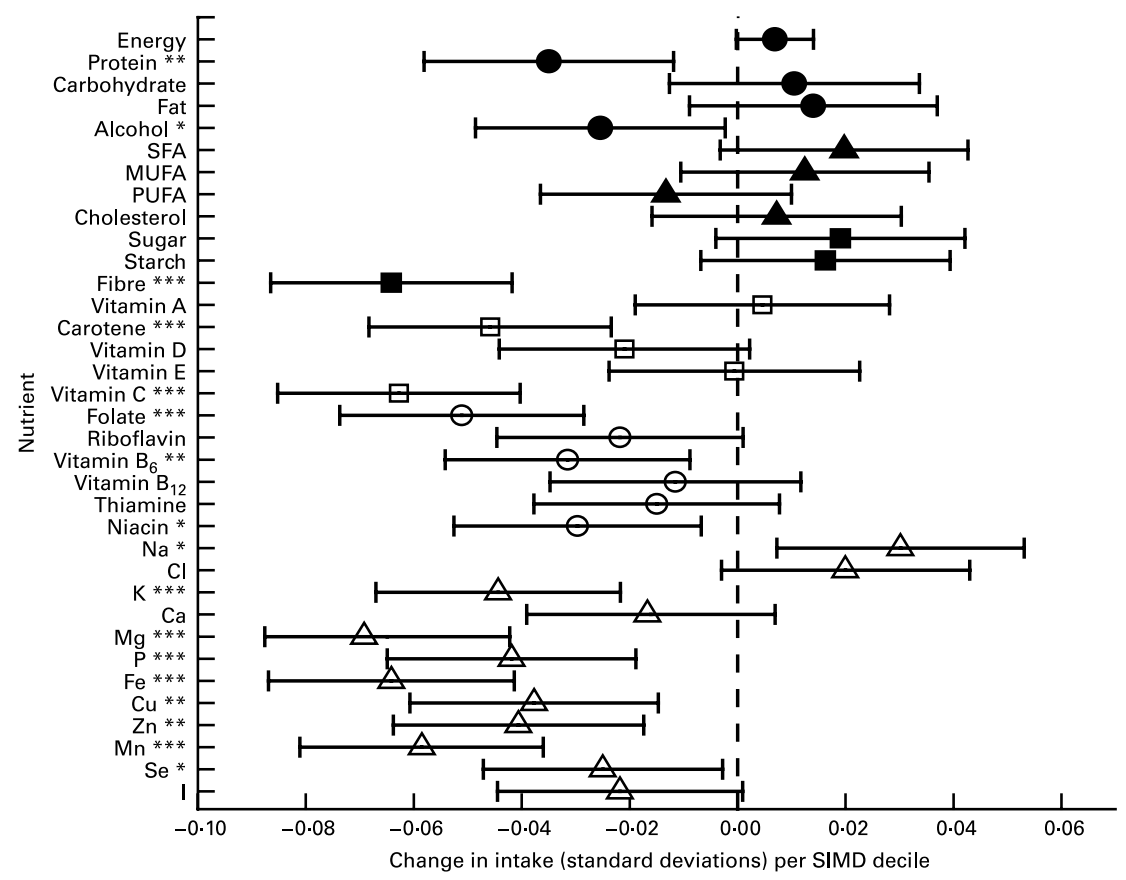

Fig. 2. Summary of regression analysis of change in nutrient intake by Scottish Index of Multiple Deprivation (SIMD). Data are presented as standard deviations of intake per deprivation decile, with $95 \% \mathrm{Cl}$ represented by horizontal bars, to allow all nutrients to be compared in one graph. Results are shown for the major macronutrient classes $(\bullet)$, fats $(\boldsymbol{\Lambda})$, carbohydrates $(\boldsymbol{\square})$, fat-soluble vitamins $(\square)$, water-soluble vitamins $(O)$, and for trace elements and minerals $(\triangle)$. The level of statistical significance for each regression is indicated beside the relevant nutrient: ${ }^{*} P<0.05,{ }^{* *} P<0.01,{ }^{\star * \star} P<0.001$. 
graphs of intake with deprivation decile (Figs. 4-6). For those nutrients patterned by deprivation there were a few, such as Fe, protein and possibly vitamin $\mathrm{C}$, where the response was approximately linear across all deprivation deciles. However, for most nutrients there was a graded change by decile in the most deprived groups with little difference in intake across the four or five least deprived deciles. This pattern was observed for folate and most of the other B vitamins, vitamin $\mathrm{C}, \beta$-carotene, dietary fibre and many of the minerals including $\mathrm{Zn}, \mathrm{Mg}$ and $\mathrm{K}$. Interestingly, the pattern for $\mathrm{Na}$ and $\mathrm{Cl}$ intake with deprivation was almost the mirror image, i.e. increasing intake with increasing deprivation, particularly in the most deprived groups. Habitual intake of folate and vitamin $B_{12}$ from foods (Fig. 6) followed essentially the same pattern by deprivation decile as the blood status measures (Fig. 1). The pattern for dietary fat was complicated, with total fat, saturated, monounsaturated and polyunsaturated fat following a biphasic relationship with deprivation decile. The generally poorer intake of essential nutrients, minerals and fibre in the more deprived women is consistent with the lower intake of fruit and vegetables, for example (Fig. 7). The graphs of food intake with deprivation decile also illustrate that, as for many of the individual nutrients, there was a differential effect with level of deprivation, with little difference in intake across the least deprived deciles but greater sensitivity to the level of deprivation in the most disadvantaged women. A number of nutrients were related to pregnancy outcome before and after controlling for deprivation index, smoking, marital status and parity (OR units are energy-adjusted dietary intake standard deviations as in Fig. 2). Births of babies in the lowest decile for standardised birth weight were most common in women consuming diets low in vitamin $\mathrm{C}$ (OR 0.79 (95\% CI $0.64,0.97) ; P=0.028$ ), riboflavin (OR 0.77 (95\% CI 0.63 ,
0.93); $P=0.008$ ), pantothenic acid (OR 0.79 (95\% CI 0.65, $0.97) ; P=0.023$ ) and sugars (OR 0.78 (95\% CI 0.64, 0.96); $P=0 \cdot 017)$. These nutrient relationships with birth weight were also significant without adjustment for deprivation index, smoking, marital status and parity. Preterm delivery was associated with diets rich in protein (OR $1.43(95 \% \mathrm{CI}$ $1.03,1.98) ; P=0.031)$ and fat (OR 1.51 (95\% CI 1.10, 2.01); $P=0 \cdot 012)$ though this relationship was only significant after adjustment for deprivation index, smoking, marital status and parity. The requirement for neonatal care was associated with higher intakes of $\mathrm{Na}$ (OR 1.18 (95\% CI 1.01, 1.38); $P=0.034), \mathrm{Cl}$ (OR 1.20 (95\% CI 1.03, 1.40); $P=0.018$ ), folate (OR 1.17 (95\% CI 1.00, 1.36); $P=0.045)$, niacin (OR $1.32(95 \%$ CI 1.14, 1.54); $P<0.001)$ and vitamin $\mathrm{C}(\mathrm{OR} 1.18$ (95\% CI 1.04, 1.37); $P=0.032$ ). With the exception of $\mathrm{Na}$ these nutrient relationships were also significant before adjustment for the deprivation index, smoking, marital status and parity. It has been suggested that Caesarean delivery is associated with a higher requirement for neonatal care, a link which was also observed in the present study, but even after adjustment for delivery type the relationship between intake of these nutrients and requirement for neonatal care remained significant; the only exception was $\mathrm{Na}(P=0.057)$.

\section{Discussion}

The consequences of adverse pregnancy outcome can be lifelong. Social inequalities in adverse pregnancy outcomes persist in the UK and actually increased between 1991 and 2000 to reach levels found 20 years previously ${ }^{(7,8,12)}$. In order to develop effective strategies to reverse this trend there is a need to identify the causal factors linking deprivation to poor pregnancy outcome. Deprivation is a complex

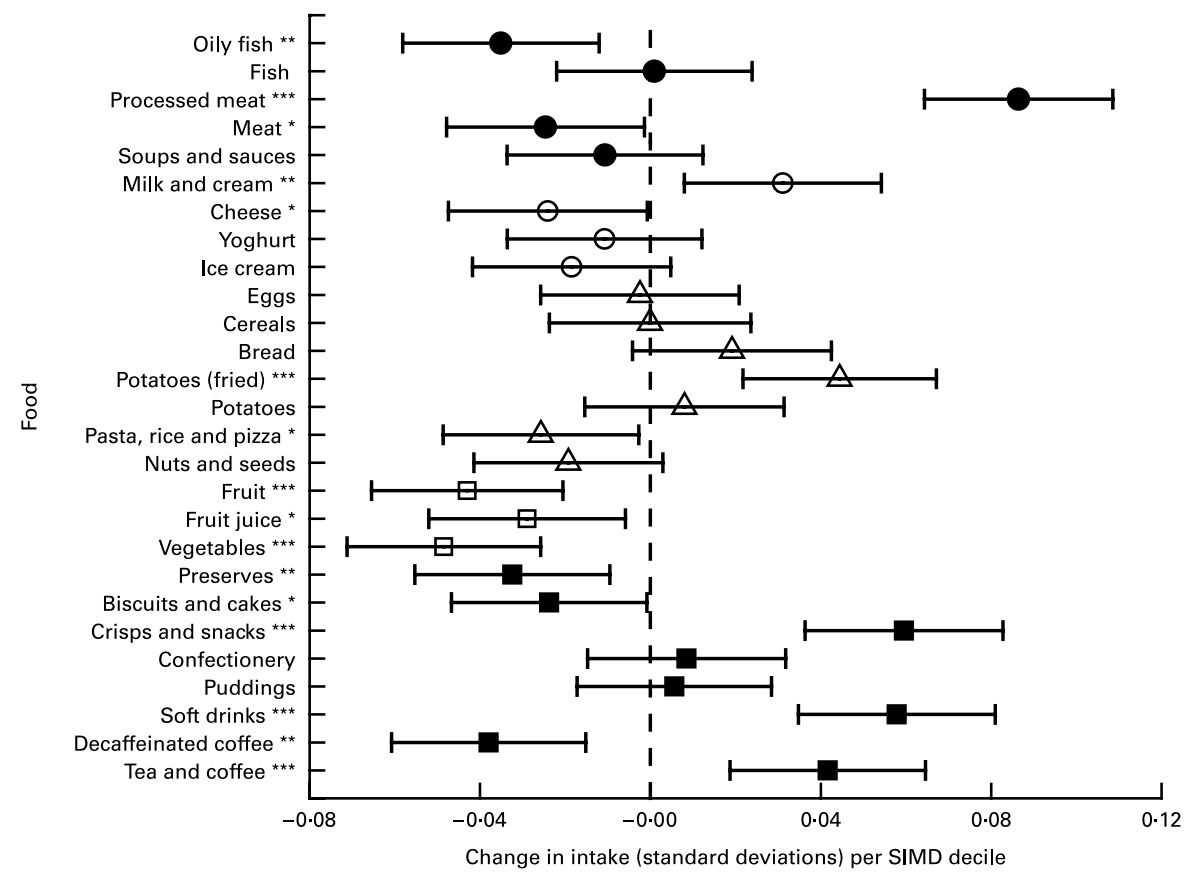

Fig. 3. Summary of regression analysis of change in intake of food types by Scottish Index of Multiple Deprivation (SIMD). Data are presented as standard deviations of intake per deprivation decile, with $95 \% \mathrm{Cl}$ represented by horizontal bars, to allow all foods to be compared in one graph. Results are shown for the main foods $(\bullet)$, dairy produce $(O)$, staples $(\triangle)$, fruit and vegetables $(\square)$, and for sweets and drinks $(\square)$. The level of statistical significance for each regression is indicated beside the relevant nutrient: ${ }^{*} P<0.05$, ${ }^{\star *} P<0.01$, ${ }^{\star \star *} P<0.001$. 

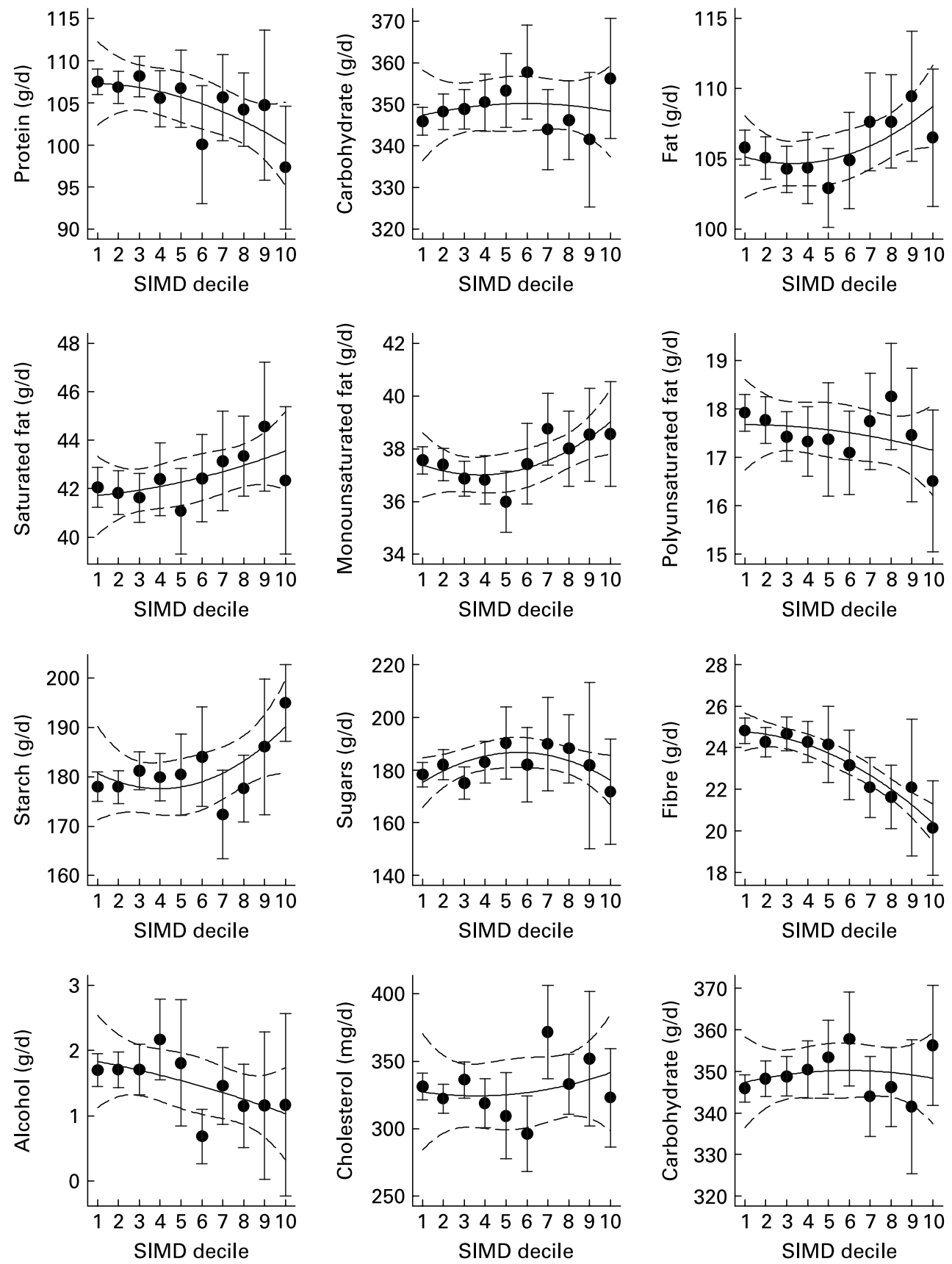

Fig. 4. Trends (polynomial fit (-) with $95 \% \mathrm{Cl}(---)$ ) by Scottish Index of Multiple Deprivation (SIMD) decile for dietary intake of the macronutrients, fibre, alcohol and cholesterol. Values are means, with $95 \% \mathrm{Cl}$ represented by vertical bars.

metric - defined by factors such as income, employment, housing, health, education, skills and training and access to services - which is also linked to behaviours, such as smoking, which are important determinants of health. Poor diet has been proposed as a key area for interventions designed to improve the health of the most deprived sector of the population.

In this population of pregnant women dietary choices were patterned by deprivation, with the most deprived women consuming diets poor in protein, fibre and a range of essential minerals such as $\mathrm{Fe}$ and vitamins such as folate, vitamin $\mathrm{B}_{6}$, niacin, vitamin $\mathrm{C}$ and $\beta$-carotene. However, the link was not observed for all nutrients and there was no evidence that intakes of vitamins $\mathrm{A}$ and $\mathrm{E}$, for example, were lower in the more deprived women. Diets in the deprived group were richer in $\mathrm{Na}$ and $\mathrm{Cl}$ - the main source of which is salt - and fat, particularly saturated and monounsaturated fat. This overall dietary pattern is generally accepted as being detrimental to health and the food choices underpinning this nutritional deprivation are consistent with much of the current advice on healthy eating being most relevant to the deprived in society. More deprived women had lower intakes of fruit, vegetables and oily fish and higher intakes of processed meat, milk and cream, crisps and snacks and soft drinks. Interestingly, the relationship between nutrient intake and deprivation 

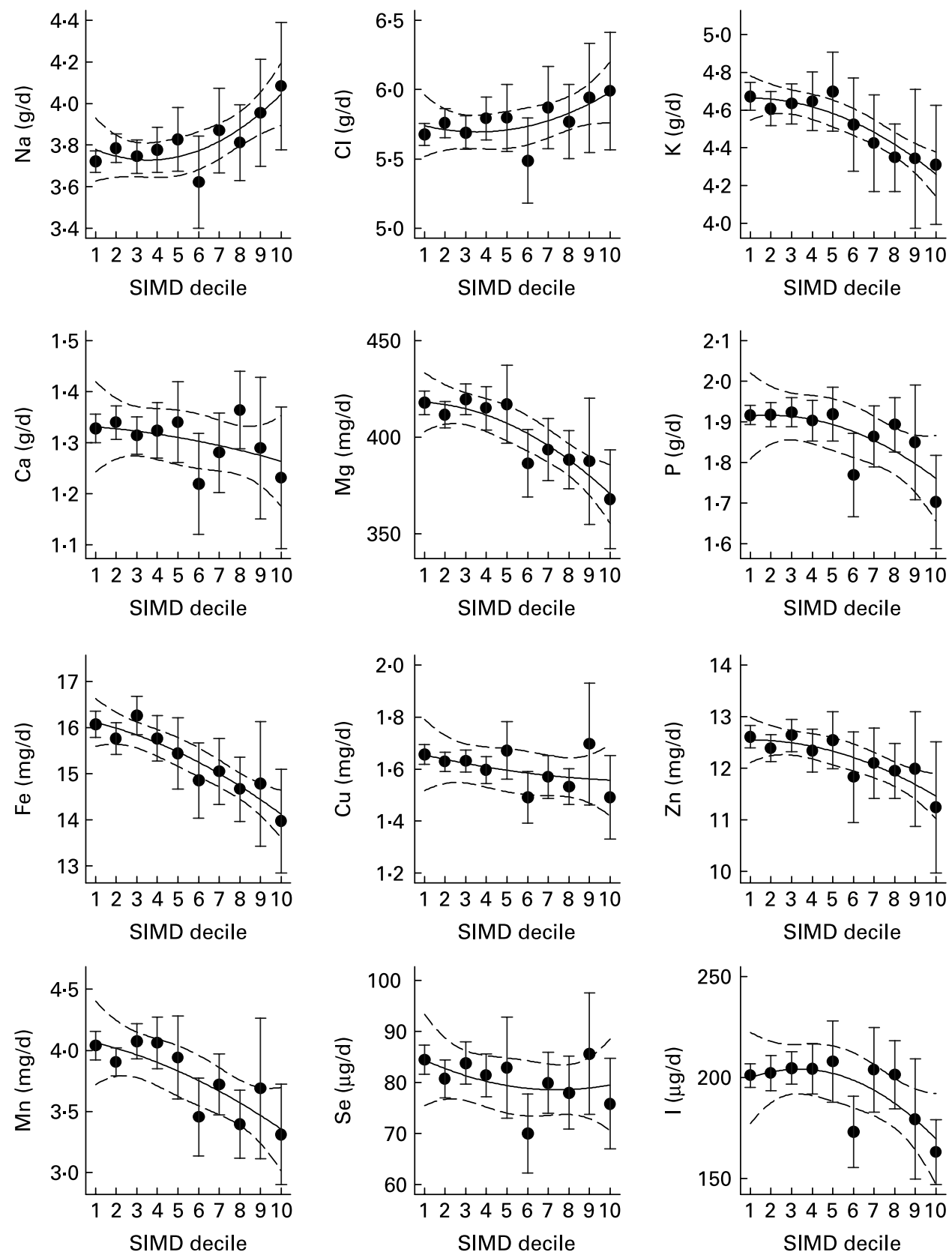

Fig. 5. Trends (polynomial fit $(-)$ with $95 \% \mathrm{Cl}(---)$ ) by Scottish Index of Multiple Deprivation (SIMD) decile for dietary intake of minerals. Values are means, with $95 \% \mathrm{Cl}$ represented by vertical bars.

was not a simple linear one. In general, there was a modest reduction in the quality of the diet with level of deprivation for the first four or five deciles but much greater dependency on the level of deprivation in the most disadvantaged in society. This deprivation-related gradient extended to supplement use. In the case of periconceptual supplementary folic acid use, the uptake by level of deprivation mirrored almost exactly the intake of folates from food. An effect of deprivation on folic acid supplement use has been reported elsewhere ${ }^{(19)}$ though that quintile-based analysis, using a less detailed index of deprivation, showed similar levels of periconceptual supplement use in quintiles 4 and 5. The strongest evidence for a beneficial effect of folic acid on neural tube defect is found in the periconceptual period, though the recommendation for supplement use extends to 12 weeks' gestation $^{(20)}$. Reported folic acid use after conception but before 12 weeks of pregnancy was high (80\%) and not different between the most and least deprived women. These data for reported folic acid use are similar to those described elsewhere $^{(19,21,22)}$. However, the strong relationship between blood folate status at 19 weeks' gestation and level of deprivation suggests that deprived women may over-report folic acid use in the period up to 12 weeks' gestation. Taken overall, these results suggest that folic acid use in the critical period is lowest in precisely those women who would benefit most because of a poor general diet. 

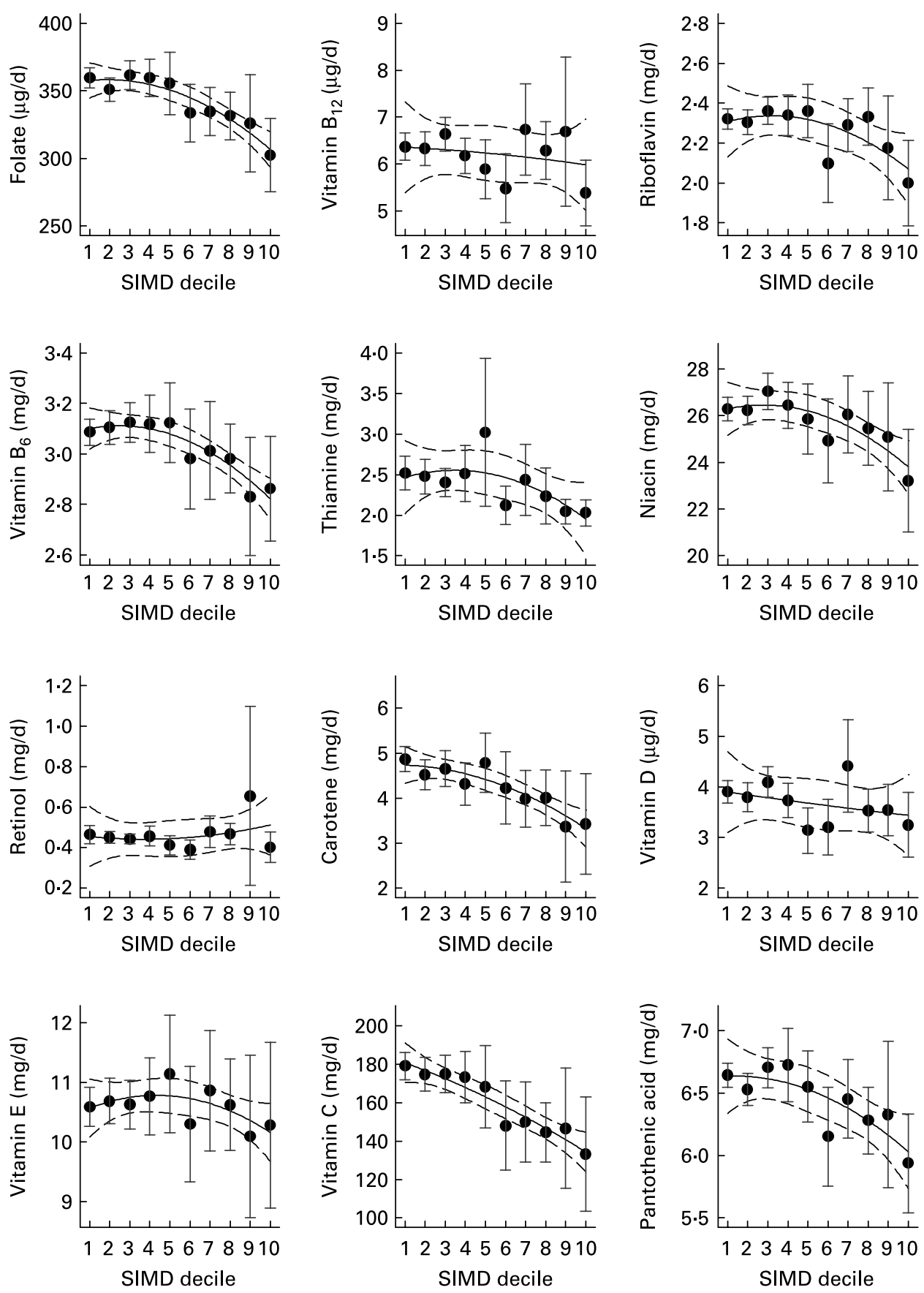

Fig. 6. Trends (polynomial fit (-) with $95 \% \mathrm{Cl}(---)$ ) by Scottish Index of Multiple Deprivation (SIMD) decile for dietary intake of vitamins. Values are means, with $95 \% \mathrm{Cl}$ represented by vertical bars.

In 1937 Boyd-Orr reported that the nutritional quality of the Scottish diet was proportional to income ${ }^{(23)}$. The data presented here for pregnant mothers suggest that this picture remains broadly true today, although the nature of deprivation has changed since 1937 . It is now defined by a range of factors including, but not limited to, income. Furthermore, increasing sophistication of food processing and the ability to manipulate the nutrient composition of highly processed foods, differential pricing of fresh and processed food, and differential access to food of high nutritional value has complicated the nature of nutritional associations with deprivation. Poor diets in the UK in the 21 st century have adequate energy but are characterised by a low density of many, but not all, of the essential nutrients. Such diets appear to contribute to inequalities in pregnancy outcomes even after adjustment for the level of deprivation.

Deprivation is related to a number of other factors that could confound the apparent link between maternal nutrition and adverse pregnancy outcome. In this population, deprivation was associated with a lower level of partner support, with the most deprived mothers being almost twice as likely to be single (not married or cohabiting) as the least deprived 

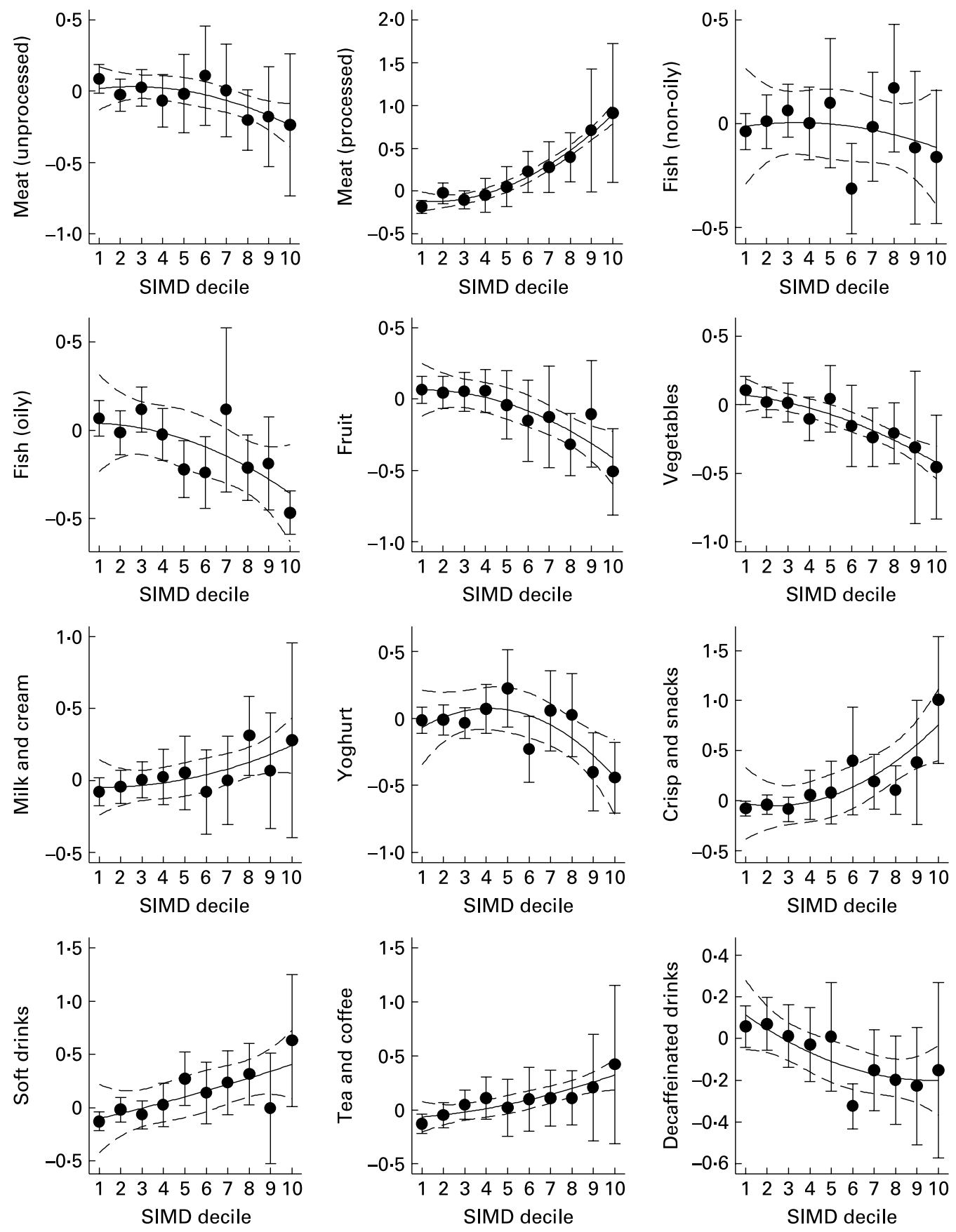

Fig. 7. Trends (polynomial fit (-) with $95 \% \mathrm{Cl}(---)$ ) by Scottish Index of Multiple Deprivation (SIMD) decile for intake of foods ( $z$-scores). Values are means with $95 \% \mathrm{Cl}$ represented by vertical bars.

mothers. Behaviours with a potential impact on health and pregnancy, such as smoking and supplement use, were also patterned by level of deprivation. A link between smoking and low birth weight has been reported elsewhere. The effect reported here persisted here even after adjustment for deprivation and a number of other potential confounders. The intake of a number of nutrients was related to birth outcome with and without adjustment for deprivation. In the case of low birth weight, the risk was highest in women with poor intakes of vitamin $\mathrm{C}$, pantothenic acid, riboflavin and sugars. Similar observations have been reported elsewhere on data that have not been adjusted for deprivation ${ }^{(24-27)}$.
For preterm birth and the need for neonatal treatment the risk was highest in women with diets high in particular nutrients - for example, protein and fat in the case of preterm birth - although this relationship was only apparent after adjustment for deprivation.

The strong relationship observed between homocysteine concentration and deprivation is consistent with data from non-pregnant populations in the USA ${ }^{(28)}$ and Europe ${ }^{(29)}$. Plasma homocysteine reflects folate intake but cannot be completely explained by nutritional factors such as folate status alone $^{(28)}$. This marker of cardiovascular health may be responsive to other non-nutritional factors linked to deprivation. 
Even after accounting for diet and other factors, such as smoking, presence of a partner, parity and age at delivery, there remained a significant relationship between deprivation and risk of preterm birth, and the need for neonatal treatment. This direct link with deprivation may be practical, possibly resulting from a higher incidence of infection or poorer uptake of obstetric services in more deprived women. Potential organic explanations include psychosocial stress and the physiological responses to stress which are thought to be patterned by social class ${ }^{(28,30)}$. Low birth weight is associated with levels of maternal stress ${ }^{(31,32)}$ and plausible biological mechanisms, involving the programming of hypothalamicpituitary-adrenal function, have been proposed to explain this $\operatorname{link}^{(33)}$

Social disadvantage in the UK persists throughout life and across generations and is proving increasingly difficult to overcome. Poor birth outcome is itself associated with lower educational attainment and disadvantage throughout life. Improving the nutrient intake of disadvantaged women of childbearing age may help to break the vicious cycle of deprivation by improving pregnancy outcome. Other factors linked to deprivation also appear to influence pregnancy outcome and it is important to also identify these if we are to break the transmission of disadvantage across the generations.

\section{Acknowledgements}

I. Fraser and A. Skene helped with recruitment, measurement of nutrient intake and laboratory analysis. The authors would like to express their gratitude to the mothers who took part in the study.

The present study was funded by the UK Food Standards Agency (grant no. N05040). P. H., S. D., G. H. and G. M. received support from the Scottish Government Rural and Environment Research and Analysis Directorate.

P. H. was responsible for study design, data collection and statistical analysis, and preparation of the manuscript. D. M. C. was responsible for study design, the clinical database and recruitment, and manuscript revision. S. D. was responsible for plasma folate and homocysteine analysis, and manuscript revision; K. A. and G. H. were responsible for sample processing and the supplement database. C. P. was responsible for erythrocyte folate analysis and manuscript revision. G. M. was responsible for study design, the dietary questionnaire and manuscript revision.

The authors report no conflict of interest in relation to the present study.

\section{References}

1. Acheson D (1998) Report of the Independent Inquiry into Inequalities in Health. London: The Stationery Office.

2. Pryor J, Silva PA \& Brooke M (1995) Growth, development and behaviour in adolescents born small-for-gestational-age. J Paediatr Child Health 31, 403-407.

3. Shenkin SD, Starr JM \& Deary IJ (2004) Birth weight and cognitive ability in childhood: a systematic review. Psychol Bull 130, 989-1013.

4. Richards M, Hardy R, Kuh D, et al. (2001) Birth weight and cognitive function in the British 1946 birth cohort: longitudinal population based study. BMJ 322, 199-203.
5. Barker DJP (1995) Fetal origins of coronary heart disease. BMJ 311, 171-174.

6. Dibben C, Sigala M \& Macfarlane A (2006) Area deprivation, individual factors and low birth weight in England: is there evidence of an 'area effect'? J Epidemiol Community Health 60, 1053-1059.

7. Fairley L (2005) Changing patterns of inequality in birthweight and its determinants: a population-based study, Scotland 1980-2000. Paediatr Perinat Epidemiol 19, 342-351.

8. Fairley L \& Leyland AH (2006) Social class inequalities in perinatal outcomes: Scotland 1980-2000. J Epidemiol Community Health 60, 31-36.

9. Gisselmann MD (2006) The influence of maternal childhood and adulthood social class on the health of the infant. Soc Sci Med 63, 1023-1033.

10. Moser K, Li L \& Power C (2003) Social inequalities in low birth weight in England and Wales: trends and implications for future population health. J Epidemiol Community Health 57, 687-691.

11. Spencer N, Bambang S, Logan S, et al. (1999) Socioeconomic status and birth weight: comparison of an area-based measure with the Registrar General's social class. J Epidemiol Community Health 53, 495-498.

12. Bartley M, Power C, Blane D, et al. (1994) Birth weight and later socioeconomic disadvantage: evidence from the 1958 British cohort study. BMJ 309, 1475-1478.

13. Attree P (2005) Low-income mothers, nutrition and health: a systematic review of qualitative evidence. Matern Child Nutr 1, 227-240.

14. Scientific Advisory Committee on Nutrition (2006) Folate and Disease Prevention. London: The Stationery Office.

15. Campbell D, Hall M, Lemon J, et al. (1993) Clinical birthweight standards for a total population in the 1980s. Br J Obstet Gynaecol 100, 436-445.

16. Scottish Index of Multiple Deprivation (2004) Data. http://www. scotland.gov.uk/Publications/2004/06/19421/38085

17. Piyathilake CJ, Macaluso M, Hine RJ, et al. (1994) Local and systemic effects of cigarette smoking on folate and vitamin B-12. Am J Clin Nutr 60, 559-566.

18. Masson LF, McNeill G, Tomany JO, et al. (2003) Statistical approaches for assessing the relative validity of a food-frequency questionnaire: use of correlation coefficients and the kappa statistic. Public Health Nutr 6, 313-321.

19. Relton CL, Hammal DM, Rankin J, et al. (2005) Folic acid supplementation and social deprivation. Public Health Nutr 8, $338-340$.

20. Committee on Medical Aspects of Food and Nutrition Policy (2002) Folic Acid and the Prevention of Disease, report no. 50. London: The Stationery Office.

21. Blake M, Herrick K \& Kelly Y (2003) Health Survey for England 2002, Maternal and Infant Health. London: The Stationery Office.

22. Hamlyn B, Brooker S, Oleinikova K, et al. (2002) Infant Feeding 2000. A Survey Conducted on Behalf of DH, the Scottish Executive, the National Assembly for Wales and DH, Social Services and Public Safety in Northern Ireland. London: The Stationery Office.

23. Boyd-Orr J (1937) Food Health and Income: A Survey of Adequacy of Diet in Relation to Income. London: Macmillan and Co. Ltd.

24. Mathews F, Yudkin P \& Neil A (1999) Influence of maternal nutrition on outcome of pregnancy: prospective cohort study. BMJ 319, 339-343.

25. Lagiou P, Mucci L, Tamimi R, et al. (2005) Micronutrient intake during pregnancy in relation to birth size. Eur $\mathbf{J}$ Nutr 44, 52-59.

26. Badart-Smook A, van Houwelingen AC, Al MD, et al. (1997) Fetal growth is associated positively with maternal intake of 
riboflavin and negatively with maternal intake of linoleic acid. $J$ Am Diet Assoc 97, 867-870.

27. Moses RG, Luebcke M, Davis WS, et al. (2006) Effect of a low-glycemic-index diet during pregnancy on obstetric outcomes. Am J Clin Nutr 84, 807-812.

28. Muennig P, Sohler N \& Mahato B (2007) Socioeconomic status as an independent predictor of physiological biomarkers of cardiovascular disease: evidence from NHANES. Prev Med 45, 35-40.

29. Panagiotakos DB, Pitsavos C, Manios Y, et al. (2005) Socio-economic status in relation to risk factors associated with cardiovascular disease, in healthy individuals from the ATTICA study. Eur J Cardiovasc Prev Rehabil 12, 68-74.
30. Adler NE \& Rehkopf DH (2007) U.S. disparities in health: description, causes, and mechanisms. Annu Rev Public Health 29, 235-252.

31. Borders AE, Grobman WA, Amsden LB, et al. (2007) Chronic stress and low birth weight neonates in a low-income population of women. Obstet Gynecol 109, 331-338.

32. Paarlberg KM, Vingerhoets AJ, Passchier J, et al. (1999) Psychosocial predictors of low birthweight: a prospective study. Br J Obstet Gynaecol 106, 834-841.

33. Meaney MJ, Szyf M \& Seckl JR (2007) Epigenetic mechanisms of perinatal programming of hypothalamic-pituitary-adrenal function and health. Trends Mol Med 13, 269-277. 